\title{
Sleep Apnea as a Cardiorenal Risk Factor in CKD and Renal Transplant Patients
}

\author{
Carmine Zoccali ${ }^{\mathrm{a}}$ Stefanos Roumeliotis ${ }^{\mathrm{b}}$ Francesca Mallamaci ${ }^{\mathrm{a}, \mathrm{c}}$ \\ ${ }^{a}$ CNR-IFC, Clinical Epidemiology of Renal Disease and Hypertension, Reggio Cal, Italy; ${ }^{\text {b}}$ Division of Nephrology \\ and Hypertension, 1st Department of Internal Medicine, School of Medicine, AHEPA Hospital, Aristotle University \\ of Thessaloniki, Thessaloniki, Greece; 'Nephrology, Dialysis and Renal Transplantation Unit, Grande Ospedale \\ Metropolitano di Reggio Cal, Reggio Cal, Italy
}

\section{Key Messages}

- In CKD patients, the prevalence of SA parallels the severity of renal dysfunction.

- SA in predialysis CKD and in ESKD patients predicts a high risk for cardiovascular disease and death.

- Volume expansion is the main factor underlying SA in these populations.

- Renal transplantation is the most effective treatment of SA in ESKD, but this disturbance re-emerges on long-term observation in these patients.

\section{Keywords}

Sleep apnea - Chronic kidney disease - End-stage kidney disease - Cardiovascular risk - Chronic kidney disease progression · Mortality

\section{Abstract \\ Background: Chronic kidney disease (CKD) is a public health priority of increasing concern worldwide. Sleep apnea (SA) of moderate-to-severe degree has a 3-9\% prevalence in women and $10-17 \%$ in men in the general population. Sum- mary: In CKD patients, the prevalence of SA parallels the de- cline of the GFR being $27 \%$ in CKD patients with a GFR of $>60$ $\mathrm{mL} / \mathrm{min} / 1.73 \mathrm{~m}^{2}$ and $57 \%$ in patients with end-stage kidney disease (ESKD). In the early CKD stages, fluid overload is probably the sole risk factor for SA in this population. At more severe CKD stages, disturbed central and peripheral chemosensitivity and the accumulation of uremic toxins might contribute to SA. Still, there is no direct evidence sup- porting this hypothesis in human studies. Observational studies coherently show that SA is a risk factor for CKD inci-}

dence and CKD progression as well as for cardiovascular disease and death in this population. However, there is no randomized clinical trial testing continuous positive airway pressure or other interventions documenting that attenuation of SA may have a favorable effect on renal and cardiovascular outcomes in CKD and ESKD patients. However, most likely, the causal nature of the association between SA and cardiorenal outcomes remains unproven. Renal transplantation is the most effective treatment of SA in patients with ESKD, but this disturbance re-emerges on long-term observation in this population. However, after renal transplantation, SA does not seem to be a predictor of adverse health outcomes.

(c) 2021 S. Karger AG, Basel

\section{Introduction}

Chronic kidney disease (CKD) is a public health priority of increasing concern [1], and projections to 2040 by the global burden of disease study rank CKD as the 5th karger@karger.com

(c) 2021 S. Karger AG, Basel

Karger $\stackrel{2}{=}$
Carmine Zoccali

Ospedali Riuniti \& CNR, Nephrology \& CNR IFC Unit Ospedali Riuniti

IT-89125 Reggio Cal (Italy)

carmine.zoccali@tin.it 


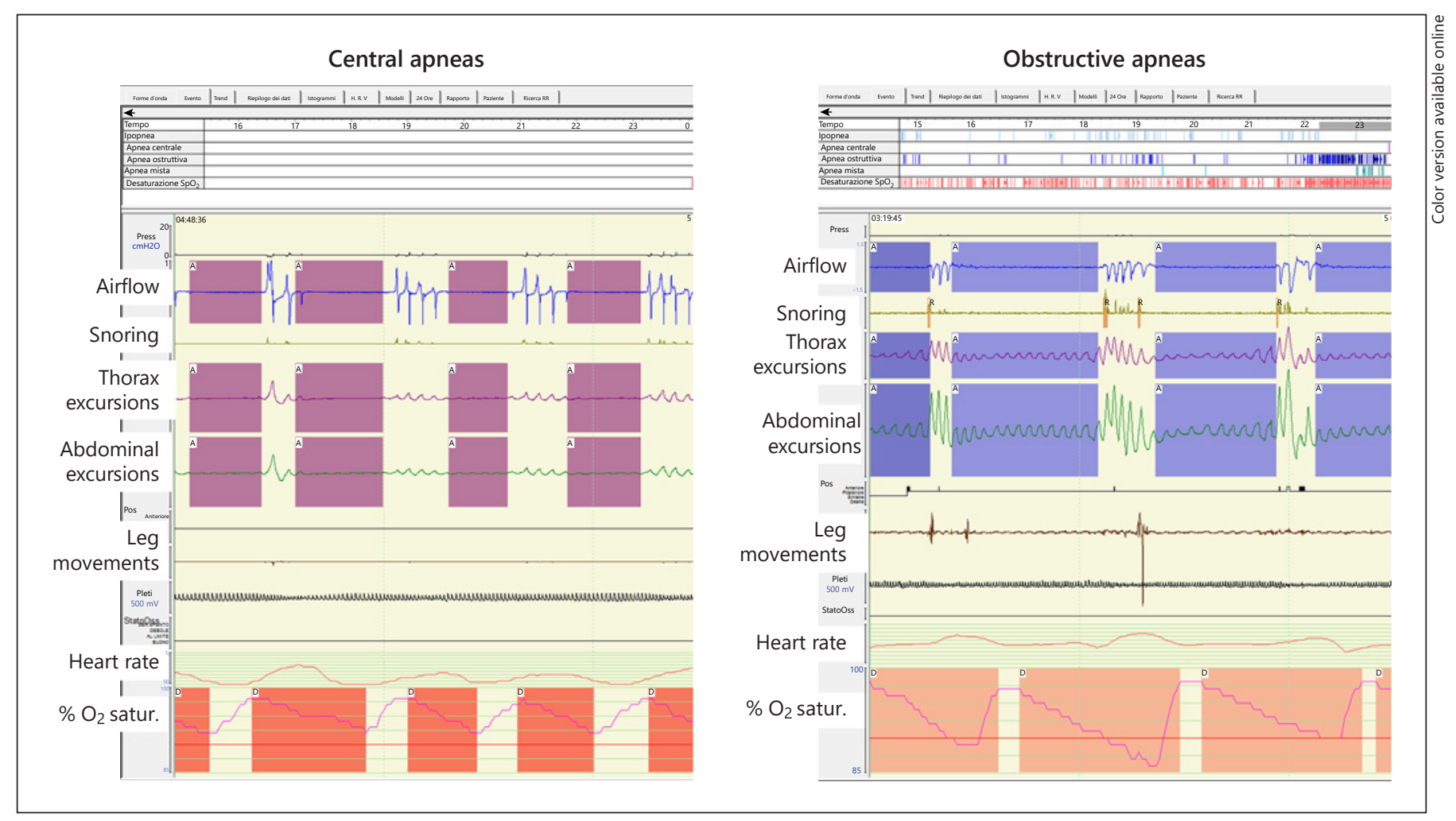

Fig. 1. Two typical examples of central and obstructive apneas. Central apnea is characterized by the lack of respiration (no airflow in the 1st trace and no thoracic and abdominal excursions in traces 3 and 4 ) and by a decline in $\mathrm{O}_{2} \%$ saturation (last trace). Obstructive apnea is characterized by a decline in $\mathrm{O}_{2} \%$ saturation simultaneous with amplified thoracic and abdominal excursions underlying a respiratory effort against an obstructed upper airway.

cause of death on a world scale [2]. Demographic and socioeconomic factors play a major role in the ascendancy of CKD among chronic noncommunicable diseases.

Sleep apnea (SA) is a condition characterized by episodes of hypoxia, sleep fragmentation, and excessive daytime sleepiness which impacts working activities and increases the risk for road traffic accidents. Studies at population level show that $3-9 \%$ of women and $10-17 \%$ of men have a moderate-to-severe SA [3]. Furthermore, SA - particularly the obstructive type - is much frequent in obesity and type 2 diabetes [4], which are the 2 most common causal risk factors for CKD [1], and sleep apnea is exceedingly frequent in the CKD population [5].

In this narrative review, we describe the SA phenotype and the pathophysiology of this condition in CKD and end-stage kidney disease (ESKD) patients. We then focus on the evidence that SA is a risk for CKD and cardiovascular disease as well as mortality in the same population. We conclude by discussing new data about the evolution of SA after renal transplantation, which is the most effective treatment of SA in ESKD.

\section{Sleep Apnea Phenotype in CKD and ESKD Patients}

In a study based on polysomnography and the relationship between renal function and SA, the prevalence of moderate-to-severe SA in CKD patients was $27 \%$ in CKD patients with a GFR of $>60 \mathrm{~mL} / \mathrm{min} / 1.73 \mathrm{~m}^{2}$ and $57 \%$ in patients with ESKD [6]. Polysomnographic studies allow the subdivision of SA into 3 types: obstructive (OSA), central (CSA), and mixed (MSA). OSA is characterized by intermittent narrowing or obstruction of the oropharynx and/or of the hypopharynx. CSA is diagnosed when there is no airway obstruction and is typified by the lack of inspiratory effort during apneic episodes (Fig. 1). OSA and CSA episodes coexist in some patients as MSA. In a metaanalysis of 8 studies by Nigam et al. [7], the prevalence of CSA among CKD patients was 9.6\%. However, this estimate was highly variable (between 0 and $75 \%$ ) among the 8 studies. In a well-designed epidemiologic survey in 6 hemodialysis centers in Switzerland [8], the prevalence of CSA was $4 \%$ which is close to an estimate (7\%) made by Beecroft in a Canadian hemodialysis unit [9]. The average 


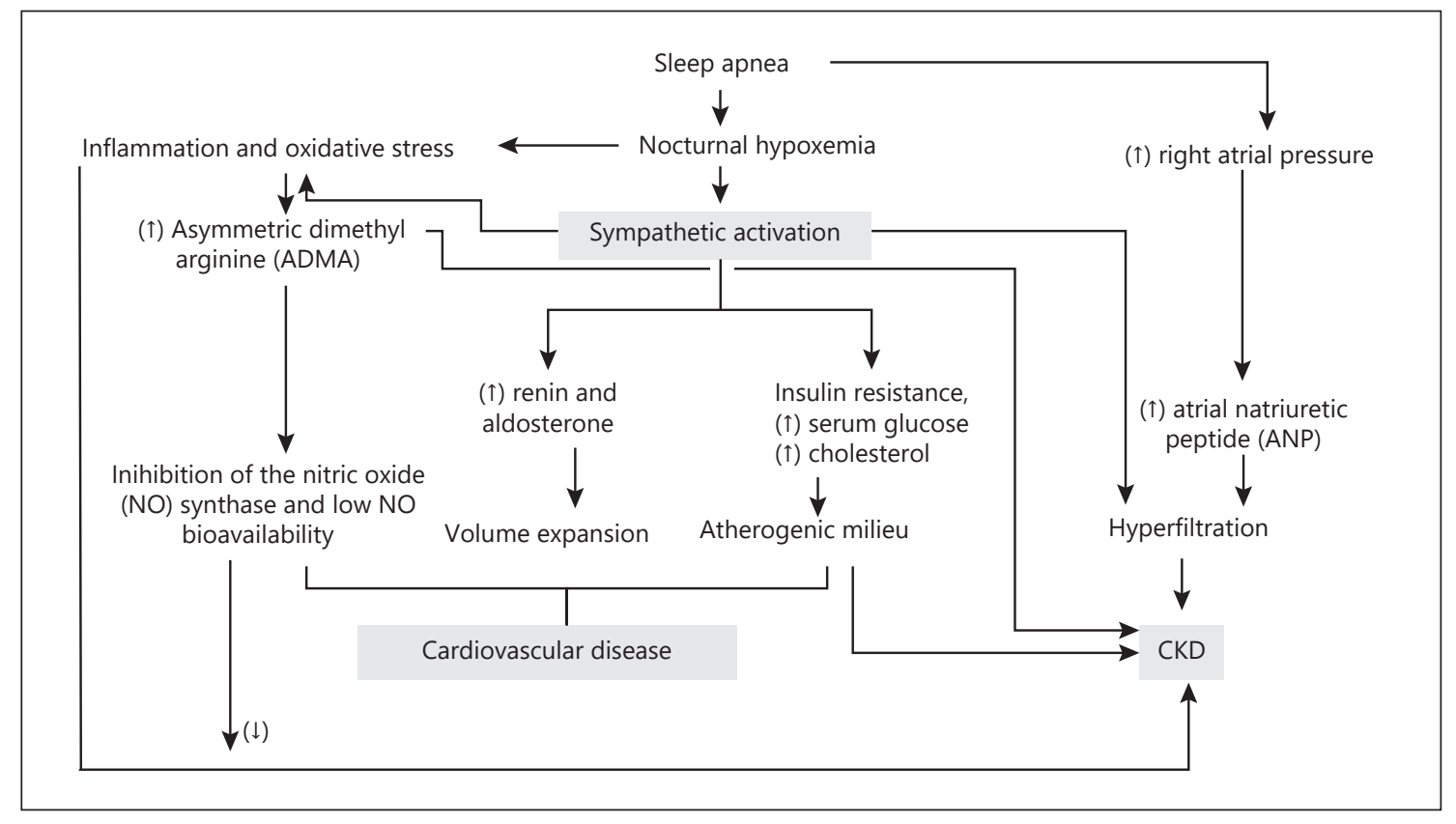

Fig. 2. Risk factors for cardiovascular disease and for CKD in sleep apnea (see main text). ( $\uparrow$ ), increase; $(\downarrow)$, decrease; NO, nitric oxide; CKD, chronic kidney disease.

number of CSA episodes in 45 hemodialysis patients in a multicenter study in Western Pennsylvania did not exceed that in a well-matched sample of the general population [10]. Overall, OSA largely outweighs CSA both among predialysis and dialysis patients.

\section{Pathophysiology and Risk Factors of Sleep Apnea in CKD and in ESKD Patients}

In the early CKD stages, fluid overload - an alteration present in about $40 \%$ of hypertensive patients with mildto-severe CKD [11] - is probably the sole risk factor for SA in this population. Fluid redistribution during supine overnight resting from the legs to the rostral parts of the body is an essential factor contributing to SA in diseases associated with volume expansion [12]. Pharyngeal and hypopharyngeal edema during sleep engenders or aggravates the obstruction of the upper airway, thereby triggering or worsening SA. Stage 1-3 CKD patients with resistant hypertension and SA manifest a significant reduction of the apnea-hypopnea index (AHI) after diuretic therapy, which goes along with the overnight change in leg fluid volume [13]. At more severe stages of CKD, other causal factors may contribute to the propensity to SA in these patients. In theory, uremic neuropathy might in- crease the collapsibility of the upper airway during sleep [14]. Increased central and peripheral chemosensitivity may destabilize respiratory control in hemodialysis patients [9]. The accumulation of uremic toxins has been implicated in SA in ESKD patients, but there is no direct evidence supporting this hypothesis. Fluid overload is more common in hemodialysis patients than in CKD patients. Thus, the relevance of fluid overload in the pathogenesis of SA in ESKD cannot be overemphasized [15, 16], and this risk factor is the sole well-established causal risk factor for SA in the ESKD population.

Repeated episodes of airway occlusion in OSA and interruption of respiration in CSA trigger cycles of hypoxemia (low $\% \mathrm{O}_{2}$ saturation) and carbon dioxide retention. Cyclical hypoxemia and hypercapnia impinge upon cardiac parasympathetic and sympathetic activity [17]. Hypoxemia increases sympathetic tone and activates the renin-angiotensin-aldosterone system. On the other hand, oxidative stress, inflammation, and hypercholesterolemia (see below) reduce nitric oxide (NO) bioavailability via the endogenous NO inhibitor asymmetric dimethylarginine [18]. These effects eventually raise blood pressure and heart rate also beyond the nocturnal sleep [17]. The prevalence of hypertension in SA is about 50 , and $83 \%$ of patients with treatment-resistant hypertension are affected by SA [19]. Sympathetic overactivity surges and the consequenc- 
es of these alterations, including hypertension, insulin resistance, and endothelial dysfunction, have multiple detrimental effects on the cardiovascular system [20] and the kidney [21]. SA impinges upon cardiovascular and renal health by an overlapping set of risk factors (Fig. 2). Furthermore, the relationship between SA and renal function is bidirectional because the eGFR is reduced among individuals with SA, particularly in those with CSA [22].

Sympathetic overactivity and its sequels, apart other relevant mechanisms in SA, are implicated in cardiovascular and renal damage. Hypoxemia augments free radical production and alters endothelial function [23]. Furthermore, oxidative stress increases vascular smooth muscle proliferation and platelet activation and aggregation [24], which generate a risky milieu for the vascular system [25] whose effects extend to the kidney [26].

As alluded to before, insulin resistance is a well-established consequence of sympathetic overactivity [27]. Insulin resistance is a hallmark in obesity and diabetes, 2 conditions also characterized by high sympathetic activity $[28,29]$. However, the link between this alteration and OSA is largely independent of obesity [30] and type 2 diabetes [31]. While the metabolic effects of insulin resistance - including hyperglycemia, dyslipidemia, and other abnormalities - are central to the proatherogenic milieu in diabetes and obesity, the same alteration in itself does not directly damage the arterial wall [32].

The effect of SA on the cardiovascular system is also mediated by the mechanical and neuroendocrine consequences of this alteration. In purely mechanical terms, excessive negative pleural and intrathoracic pressures during inspiration against an obstructed upper airway reduce right atrial pressure which, in turn, augments venous return to the heart [33]. Because the function of the 2 ventricles is strictly interrelated, the increase in right ventricular end-diastolic volume by the increased venous return determines a reduction in left ventricular stroke volume. On the other hand, excessive reduction in intrathoracic pressure during inspiration increases ventricular transmural pressure and consequently ventricular afterload [34]. Repeated episodes of nocturnal hypoxemia by OSA and CSA cause pulmonary vasoconstriction and arrhythmia and reduce by a direct mechanism of ventricular contractility and diastolic relaxation [27]. High aldosterone secondary to the activation of the renin-angiotensin system triggered by sympathetic overactivity, the increased left ventricular afterload, and wall stress, coronary heart disease and the amplification of the risk of myocardial infarction [35], and pulmonary vasoconstriction all concur to more than double the risk for heart failure in SA [36].

\section{Sleep Apnea as a Cardiorenal Risk Factor}

Heart failure, coronary heart disease, and arrhythmia [37] are the main factors underlying the high risk for cardiovascular events and death, including sudden death, in patients with SA [38]. Furthermore, independently of hypertension and other risk factors, SA per se doubles the risk for incident stroke [39]. Even though the association between SA and the risk for cardiovascular events is robust, the causal nature of these associations remains uncertain. A recent meta-analysis of intervention trials in patients with SA failed to show a benefit of continuous positive airway pressure (CPAP) on the incidence of these events [40]. However, to adequately investigate this fundamental question, large, long-term studies warranting adequate CPAP adherence are needed, which makes such studies very hard to perform.

As alluded to before, SA is traditionally held as a risk factor for incident CKD and CKD progression [41]. Full et al. [42] have recently re-examined the incident risk for CKD by SA in the 1,525 participants in the Atherosclerosis Risk in Communities (ARIC) study who completed in-home polysomnography assessments. In this study, during 19 years of follow-up, the presence of severe OSA adjusted for demographics and lifestyle behaviors predicted a $51 \%$ excess risk of incident CKD (stage 3 or higher). However, this excess risk attenuated to a mere, nonsignificant, $7 \%$ after adjustment for body mass index. On the other hand, in a Canadian study in 858 adults referred for diagnostic testing for SA, the presence of nocturnal hypoxemia predicted a 2.89 higher risk of accelerated renal function loss after adjustment for potential confounders, including the BMI. In chronic obstructive pulmonary disease, hypoxemia goes along with glomerular hyperfiltration [43]. In 2 relatively small studies in OSA patients, nocturnal hypoxemia associated with hyperfiltration and CPAP improved this alteration $[44,45]$. A recent meta-analysis of 6 cross-sectional studies and 2 retrospective case-control studies, including 8,795 participants, underlined the strong association between OSA and CKD [5]. However, the nature of this link (causal vs. noncausal) remains uncertain. Obesity is a strong confounder for the interpretation of the incident risk of CKD by OSA in the general population [42]. Studies investigating the relationship between OSA and CKD progression rate are too small to allow definitive conclusions. Until now, just 1 randomized clinical trial tested the effect of CPAP on renal function, a substudy of the SAVE (Sleep Apnea Cardiovascular Endpoints) trial [46]. In this substudy, CPAP did not modify the rate of 
eGFR loss or the urinary albumin-to-creatinine ratio. Furthermore, CPAP adherence did not influence either of these renal outcomes.

\section{The Risk of Death and Cardiovascular Events of Sleep Apnea in CKD and ESKD Patients}

The first study describing a link between SA as measured by nocturnal pulse oximetry and cardiovascular events in ESKD patients is now 20 years old [47]. Nocturnal hypoxemia alters the circadian BP profile [48] and associates with altered sympathovagal balance [49] and concentric left ventricular hypertrophy [50] in ESKD. Very sparse evidence, summarized in a meta-analysis by Tang et al. [51], shows that patients with CKD and various sleep disorders (including SA, periodic limb movement, or altered sleep duration/quality) have a $47 \%$ excess risk of death. In a recent cohort study based on polysomnography in 180 stage $4-5$ CKD patients (92 on dialysis), nocturnal hypoxemia, but not the AHI, associated with the risk of death independently of other risk factors, including the BMI [52]. In a study in 558 incident hemodialysis patients (average age, 56 years) in the Baltimore area, past diagnosis of SA was associated with a higher risk of sudden death in analyses adjusted for demographics factors, comorbidities, and BMI but not with all-cause or cardiovascular mortality. In contrast, an extensive study in elderly patients (average age, 77 years) with a past diagnosis of SA initiating dialysis in the USA $(n=15,121)$, SA was weakly associated with lower, rather than higher, risk of death and cardiovascular events [53]. A weakness of these 2 studies based on administrative databases is the fact that the past diagnosis of SA was not confirmed with a new polysomnographic study after starting regular dialysis treatment. The causal nature of the association between SA and mortality and cardiovascular disease in predialysis CKD and ESKD patients remains much uncertain because no randomized clinical trial tested the effect of interventions targeting SA on these outcomes in this population.

\section{Treatment of Sleep Apnea in CKD and ESKD Patients and the Evolution of Sleep Apnea after Renal Transplantation}

CPAP is the golden standard treatment of SA. As discussed, treatment of fluid overload mitigates SA in stage 1-3 CKD patients with SA and resistant hypertension
[13] and the association between rostral volume shift has been confirmed in hemodialysis patients [16]. Of note, long nocturnal hemodialysis [54] and long, cycler-assisted, nocturnal peritoneal dialysis [51] - two treatments that facilitate fluid overload removal - both improve SA in ESKD patients. Restored renal function after transplantation corrects fluid overload, uremic toxicity, and other factors. However, at 5 years posttransplantation, about $1 / 3$ of renal transplant patients are again volume expanded [55], and there is a surge of obesity posttransplantation [56]. A recent long-term longitudinal study registered a progressive worsening of SA, which went along with the increase in BMI in renal transplant patients [57]. Furthermore, analyses in the database of the same study showed that independently of Framingham risk factors and the BMI and the GFR, repeated measures of the AHI were directly associated with 24-h, daytime and nighttime systolic BP. Thus, SA is per se a risk factor for hypertension in renal transplant patients [58].

Lubas et al. [59] investigated the relationship between SA and graft survival time and death with graft loss in a historical cohort, single-center study of 322 renal transplant patients who lost their graft or died with a functioning graft during follow-up. In patients transplanted between 2009 and 2017, SA was associated with a 2.61 higher risk of a composite endpoint (graft loss or cardiovascular-related death with a functioning graft). In contrast, an opposite association (a 37\% risk reduction) was observed during the first decade of observation (19972008). Misclassification of SA during the first decade was very likely, and therefore, this study remains substantially inconclusive. In a study in 822 renal transplant patients based on the Berlin questionnaire, a weak surrogate marker of SA [60], a high score of this questionnaire predicted graft loss in women but not in men [61]. Another cohort study, including 100 renal transplant recipients, found no association between SA and adverse clinical outcomes, including mortality and the rate of GFR loss [62]. In another study, including 404 renal transplant patients with a pretransplantation diagnosis of SA and 117 with a de novo diagnosis of SA posttransplantation, this alteration failed to predict mortality and graft loss [63]. Renal denervation mitigates OSA [64], but no study has tested the effect of this intervention on SA in CKD and in ESKD patients.

For this reason, the apparent lack of harmful effects of SA posttransplantation has been attributed to the denervation of the transplanted kidneys $[62,63]$ or a highly hypothetic ischemic preconditioning effect of apneic episodes [63]. Overall, the critical question of whether SA 
predicts adverse clinical outcomes in renal transplant patients needs extensive, well-designed, long-term cohort studies based on polysomnography. Whether SA is harmful in renal transplant patients remains an unresolved question.

\section{Conclusions}

In CKD patients, the prevalence of SA parallels the severity of renal dysfunction and predicts a high risk for cardiovascular disease and death. Volume expansion is the main factor underlying SA in this population. Renal transplantation is the most effective treatment of SA in ESKD, but this disturbance re-emerges on long-term observation in these patients.

\section{Conflict of Interest Statement}

The authors declare no conflicts of interest.

\section{Funding Sources}

The authors did not receive any funding.

\section{Author Contributions}

Carmine Zoccali planned the review and made the literature search. He wrote the first version of the review. The other authors added additional scientific information and gave suggestions for improving the clarity of the manuscript. All authors approved the final version of the manuscript.

\section{References}

1 Bikbov B, Purcell CA, Levey AS, Smith M, Abdoli A, Abebe M, et al. Global, regional, and national burden of chronic kidney disease, 1990-2017: a systematic analysis for the Global Burden of Disease Study 2017. Lancet. 2020 Feb 29;395:709-33.

2 Foreman KJ, Marquez N, Dolgert A, Fukutaki K, Fullman N, McGaughey M, et al. Forecasting life expectancy, years of life lost, and allcause and cause-specific mortality for 250 causes of death: reference and alternative scenarios for 2016-40 for 195 countries and territories. Lancet. 2018 Nov 10;392:2052-90.

3 Peppard PE, Young T, Barnet JH, Palta M, Hagen EW. Hla KM. Increased prevalence of sleep-disordered breathing in adults. Am J Epidemiol. 2013;177(9):1006-14.

4 Jehan S, Myers AK, Zizi F, Pandi Perumal SR, Louis GJ, Mc Farlane SI. Obesity, obstructive sleep apnea and type 2 diabetes mellitus: epidemiology and pathophysiologic insights. Sleep Med Disord Int J. 2018 Jun 21;2(3):52.

5 Umbro I, Fabiani V, Fabiani M, Angelico F, Del Ben M. A systematic review on the association between obstructive sleep apnea and chronic kidney disease. Sleep Med Rev. 2020; 53:101337.

6 Nicholl DDM, Ahmed SB, Loewen AHS, Hemmelgarn BR, Sola DY, Beecroft JM, et al. Declining kidney function increases the prevalence of sleep apnea and nocturnal hypoxia. Chest. 2012;141(6): 1422.

7 Nigam G, Pathak C, Riaz M. A systematic review of central sleep apnea in adult patients with chronic kidney disease. Sleep Breath. 2016 Sep 1;20(3):957-64.

8 Forni Ogna V, Ogna A, Pruijm M, Bassi I, Zuercher E, Halabi G, et al. Prevalence and diagnostic approach to sleep apnea in hemodialysis patients: a population study. Biomed Res Int. 2015;2015:103686-9.
9 Beecroft J, Duffin J, Pierratos A, Chan CT, McFarlane P, Hanly PJ. Enhanced chemo-responsiveness in patients with sleep apnoea and end-stage renal disease. Eur Respir J. 2006 Jul 1;28(1):151-8.

10 Unruh ML, Sanders MH, Redline S, Piraino BM, Umans JG, Hammond TC, et al. Sleep apnea in patients on conventional thriceweekly hemodialysis: comparison with matched controls from the Sleep Heart Health Study. J Am Soc Nephrol. 2006;17(12):35039.

11 Braam B, Lai CF, Abinader J, Bello AK. Extracellular fluid volume expansion, arterial stiffness and uncontrolled hypertension in patients with chronic kidney disease. Nephrol Dial Transplant. 2020 Aug 1;35(8): 1393-8.

12 White LH, Bradley TD. Role of nocturnal rostral fluid shift in the pathogenesis of obstructive and central sleep apnoea. J Physiol. 2013 Mar;591(5):1179-93.

13 Kasai T, Bradley TD, Friedman O, Logan AG. Effect of intensified diuretic therapy on overnight rostral fluid shift and obstructive sleep apnoea in patients with uncontrolled hypertension. J Hypertens. 2014;32(3):673-80.

14 Tarasuik A, Heimer D, Bark H. Effect of chronic renal failure on skeletal and diaphragmatic muscle contraction. Am Rev Respir Dis. 1992 Dec 17;146(6):1383-8.

15 Lyons OD, Inami T, Perger E, Yadollahi A, Chan CT, Bradley TD. The effect of fluid overload on sleep apnoea severity in haemodialysis patients. Eur Respir J. 2017 Apr 1; 49(4):49.

16 Ogna A, Forni Ogna V, Mihalache A, Pruijm M, Halabi G, Phan O, et al. Obstructive sleep apnea severity and overnight body fluid shift before and after hemodialysis. Clin J Am Soc Nephrol. 2015;10(6):1002-10.
17 Kim JB, Seo BS, Kim JH. Effect of arousal on sympathetic overactivity in patients with obstructive sleep apnea. Sleep Med. 2019 Oct 1; 62:86-91.

18 Badran M, Golbidi S, Ayas N, Laher I. Nitric oxide bioavailability in obstructive sleep apnea: interplay of asymmetric dimethylarginine and free radicals. Sleep Disord. 2015; 2015:387801-10.

19 Logan AG, Perlikowski SM, Mente A, Tisler A, Tkacova R, Niroumand M, et al. High prevalence of unrecognized sleep apnoea in drugresistant hypertension. J Hypertens. 2001; 19(12):2271-7.

20 Malpas SC. Sympathetic nervous system overactivity and its role in the development of cardiovascular disease. Physiol Rev. 2010 Apr; 90(2):513-57.

21 Kaur J, Young BE, Fadel PJ. Sympathetic overactivity in chronic kidney disease: consequences and mechanisms. Int J Mol Sci. 2017 Aug 2;18(8):18

22 Fleischmann G, Fillafer G, Matterer H, Skrabal F, Kotanko P. Prevalence of chronic kidney disease in patients with suspected sleep apnoea. Nephrol Dial Transplant. 2010 Jan; 25(1):181-6

23 Lavie L. Oxidative stress: a unifying paradigm in obstructive sleep apnea and comorbidities. Prog Cardiovasc Dis. 2009;51(4):303-12.

24 Freedman JE. Oxidative stress and platelets. Arterioscler Thromb Vasc Biol. 2008 Mar 1; 28(3):s11.

25 Lévy P, Pépin JL, Arnaud C, Baguet JP, Dematteis M. Mach F. Obstructive sleep apnea and atherosclerosis. Prog Cardiovasc Dis. 2009 Mar;51:400-10.

26 Poonit ND, Cai XH. The relation and mechanism of kidney injury in obstructive sleep apnea: a literature review. Sleep Biol Rhythms. 2018;16:151-67. 
27 Gomez A, Mink S. Interaction between effects of hypoxia and hypercapnia on altering left ventricular relaxation and chamber stiffness in dogs. Am Rev Respir Dis. 1992;146(2):313.

28 Grassi G, Biffi A, Seravalle G, Trevano FQ, Dell'oro R, Corrao G, et al. Sympathetic neural overdrive in the obese and overweight state: meta-analysis of published studies. Hypertension. 2019 Aug 1;74:349-58.

29 Grassi G, Biffi A, Dell'Oro R, Quarti Trevano F, Seravalle G, Corrao G, et al. Sympathetic neural abnormalities in type 1 and type 2 diabetes: a systematic review and meta-analysis. J Hypertens. 2020 Aug 1;38(8):1436-42.

30 Punjabi NM, Sorkin JD, Katzel LI, Goldberg AP, Schwartz AR, Smith PL. Sleep-disordered breathing and insulin resistance in middleaged and overweight men. Am J Respir Crit Care Med. 2002 Mar;165(5):677-82.

31 Aronsohn RS, Whitmore H, Van Cauter E, Tasali E. Impact of untreated obstructive sleep apnea on glucose control in type 2 diabetes. Am J Respir Crit Care Med. 2010;181(5):507.

32 Semenkovich CF. Insulin resistance and atherosclerosis. J Clin Invest. 2006 Jul 3;116(7): 1813-22.

33 Jellinek H, Krenn H, Oczenski W, Veit F, Schwarz S, Fitzgerald RD. Influence of positive airway pressure on the pressure gradient for venous return in humans. J Appl Physiol. 2000;88(3):926.

34 Marrone O, Bellia V, Ferrara G, Milone F, Romano L, Salvaggio A, et al. Transmural pressure measurements. Importance in the assessment of pulmonary hypertension in obstructive sleep apneas. Chest. 1989;95(2):338.

35 Marin JM, Carrizo SJ, Vicente E, Agusti AG. Long-term cardiovascular outcomes in men with obstructive sleep apnoea-hypopnoea with or without treatment with continuous positive airway pressure: an observational study. Lancet. 2005 Mar;365(9464):1046-53.

36 Shahar E, Whitney CW, Redline S, Lee ET, Newman AB, Nieto FJ, et al. Sleep-disordered breathing and cardiovascular disease: crosssectional results of the Sleep Heart Health Study. Am J Respir Crit Care Med. 2001; 163(1):19-25.

37 Mehra R, Benjamin EJ, Shahar E, Gottlieb DJ, Nawabit R, Kirchner HL, et al. Association of nocturnal arrhythmias with sleep-disordered breathing: the Sleep Heart Health Study. Am J Respir Crit Care Med. 2006;173(8):910.

38 Kohli P, Balachandran JS, Malhotra A. Obstructive sleep apnea and the risk for cardiovascular disease. Curr Atheroscler Rep. 2011 Apr;13(2):138-46.

39 McDermott M, Brown DL. Sleep apnea and stroke. Curr Opin Neurol. 2020 Feb 1;33(1): 4-9.

40 Labarca G, Dreyse J, Drake L, Jorquera J, Barbe F. Efficacy of continuous positive airway pressure (CPAP) in the prevention of cardiovascular events in patients with obstructive sleep apnea: systematic review and metaanalysis. Sleep Med Rev. 2020 Aug 1;52: 101312.
41 Hanly PJ, Ahmed SB. Sleep apnea and the kidney: is sleep apnea a risk factor for chronic kidney disease? Chest. 2014 Oct;146(4):111422.

42 Full KM, Jackson CL, Rebholz CM, Matsushita K, Lutsey PL. Obstructive sleep apnea, other sleep characteristics, and risk of CKD in the atherosclerosis risk in communities Sleep Heart Health Study. J Am Soc Nephrol. 2020 Aug;31(8):1859-69.

43 Bratel T, Ljungman S, Runold M, Stenvinkel $P$. Renal function in hypoxaemic chronic obstructive pulmonary disease: effects of longterm oxygen treatment. Respir Med. $2003 \mathrm{Apr}$ 1;97(4):308-16

44 Nicholl DD, Hanly PJ, Poulin MJ, Handley GB, Hemmelgarn BR, Sola DY, et al. Evaluation of continuous positive airway pressure therapy on renin-angiotensin system activity in obstructive sleep apnea. Am J Respir Crit Care Med. 2014 Sep 1;190(5):572-80.

45 Kinebuchi S, Kazama JJ, Satoh M, Sakai K, Nakayama H, Yoshizawa H, et al. Short-term use of continuous positive airway pressure ameliorates glomerular hyperfiltration in patients with obstructive sleep apnoea syndrome. Clin Sci. 2004 Sep 1;107(3):317-22.

46 Loffler KA, Heeley E, Freed R, Anderson CS, Brockway B, Corbett A, et al. Effect of obstructive sleep apnea treatment on renal function in patients with cardiovascular disease. Am J Respir Crit Care Med. 2017 Dec 1;196: 1456-62.

47 Zoccali C, Mallamaci F, Tripepi G. Nocturnal hypoxemia predicts incident cardiovascular complications in dialysis patients. J Am Soc Nephrol. 2002 Mar;13(3):729-33.

48 Zoccali C, Benedetto FA, Tripepi G, Cambareri F, Panuccio V, Candela V, et al. Nocturnal hypoxemia, night-day arterial pressure changes and left ventricular geometry in dialysis patients. Kidney Int. 1998 Apr;53(4): 1078-84.

49 Zoccali C, Mallamaci F, Tripepi G, Benedetto FA. Autonomic neuropathy is linked to nocturnal hypoxaemia and to concentric hypertrophy and remodelling in dialysis patients. Nephrol Dial Transplant. 2001;16(1):70-7.

50 Zoccali C, Benedetto FA, Mallamaci F, Tripepi G, Candela V, Labate C, et al. Left ventricular hypertrophy and nocturnal hypoxemia in hemodialysis patients. J Hypertens. $2001 \mathrm{Feb} ; 19(2): 287-93$.

51 Tang SC, Lam B, Ku PP, Leung WS, Chu CM, Ho YW, et al. Alleviation of sleep apnea in patients with chronic renal failure by nocturnal cycler-assisted peritoneal dialysis compared with conventional continuous ambulatory peritoneal dialysis. J Am Soc Nephrol. 2006 Sep;17(9):2607-16.

52 Jhamb M, Ran X, Abdalla H, Roumelioti ME, Hou S, Davis H, et al. Association of sleep apnea with mortality in patients with advanced kidney disease. Clin J Am Soc Nephrol. 2020 Feb 7;15(2):182-90.
53 Tuohy CV, Montez-Rath ME, Turakhia M, Chang TI, Winkelman JW, Winkelmayer WC. Sleep disordered breathing and cardiovascular risk in older patients initiating dialysis in the United States: a retrospective observational study using medicare data. BMC Nephrol. 2016;17:16.

54 Hanly PJ, Pierratos A. Improvement of sleep apnea in patients with chronic renal failure who undergo nocturnal hemodialysis. N Engl J Med. 2001 Jan 11;344(2):102-7.

55 Chan W, Bosch JA, Jones D, McTernan PG, Inston $\mathrm{N}$, Moore $\mathrm{S}$, et al. Hypervolemia and blood pressure in prevalent kidney transplant recipients. Transplantation. 2014 Aug 15; 98(3):320-7.

56 Ahmadi SF, Zahmatkesh G, Streja E, Molnar MZ, Rhee CM, Kovesdy CP, et al. Body mass index and mortality in kidney transplant recipients: a systematic review and meta-analysis. Am J Nephrol. 2014 May 22;40(4):315-24.

57 Mallamaci F, Tripepi R, D’Arrigo G, Porto G, Versace MC, Marino C, et al. Long-term changes in sleep disordered breathing in renal transplant patients: relevance of the BMI. J Clin Med. 2020 Jun 4;9(6):1739.

58 Mallamaci F, Tripepi R, D’Arrigo G, Panuccio V, Parlongo G, Caridi G, et al. Sleep-disordered breathing and 24-hour ambulatory blood pressure monitoring in renal transplant patients: longitudinal study. J Am Heart Assoc. 2020 Jul 7;9(13):e016237.

59 Lubas MM, Ware JC, Szklo-Coxe M. Sleep apnea and kidney transplant outcomes: findings from a 20-year (1997-2017) historical cohort study. Sleep Med. 2019 Nov;63:151-8.

60 Kapur VK, Auckley DH, Chowdhuri S, Kuhlmann DC, Mehra R, Ramar K, et al. Clinical practice guideline for diagnostic testing for adult obstructive sleep apnea: an American Academy of Sleep Medicine clinical practice guideline. J Clin Sleep Med. 2017 Mar 15; 13(3):479-504.

61 Szentkiralyi A, Czira ME, Molnar MZ, Kovesdy CP, Remport A, Szeifert L, et al. High risk of obstructive sleep apnea is a risk factor of death censored graft loss in kidney transplant recipients: an observational cohort study. Sleep Med. 2011 Mar;12(3):267-73.

62 Fornadi K, Ronai KZ, Turanyi CZ, Malavade TS, Shapiro CM, Novak M, et al. Sleep apnea is not associated with worse outcomes in kidney transplant recipients. Sci Rep. 2014 Nov 11;4:6987.

63 Tiwari R, Lyu B, Alagusundaramoorthy S, Astor BC, Mandelbrot DA, Parajuli S. Association of diagnosed obstructive sleep apnea with kidney transplant outcomes. Clin Transplant. 2019 Dec 26;33(12).

64 Witkowski A, Prejbisz A, Florczak E, Kądziela J, Śliwiński P, Bieleń P, et al. Effects of renal sympathetic denervation on blood pressure, sleep apnea course, and glycemic control in patients with resistant hypertension and sleep apnea. Hypertension. 2011 Oct;58(4):559-65. 